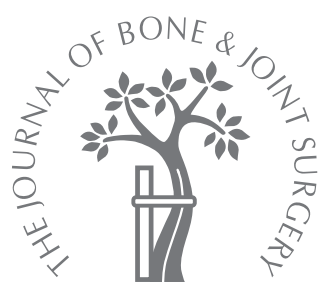

S. E. Mathew, V. Madhuri, M. Alexander, N. M. Walter, S. V. Gibikote

From Christian Medical College, Vellore, India

S. E. Mathew, MBBS, DOrth, Registrar

V. Madhuri, MS(Orth), MCh, Professor

Paediatric Orthopaedic Unit - M. Alexander, MD(Path), Assistant Professor

N. M. Walter, MD, FRCPath, Professor

Department of Pathology

I. V. Gibikote, DMRD,

DNB(Radiology), Professor

Department of Radiology

Christian Medical College

Vellore, Tamil Nadu 632004

India.

Correspondence should be sent to Professor V. Madhuri; e-mail: madhuriwalter@cmcvellore.ac.in

(C)2011 British Editorial Society of Bone and Joint Surgery doi:10.1302/0301-620X.93B3. $24955 \$ 2.00$

$J$ Bone Joint Surg [Br] 2011;93-B:418-20. Received 3 April 2010; Accepted after revision 15 November 2010

\title{
Florid reactive periostitis of the forearm bones in a child
}

\begin{abstract}
Florid reactive periostitis is a pronounced periosteal reaction, usually affecting the hands and feet, for which there is no obvious cause. It is rare in children and in long bones. We report an unusual case of florid reactive periostitis in a ten-year-old girl that involved both bones of the forearm. The lesion resolved over a period of one year, leaving a residual exostosis. She developed a physeal bar in the distal ulna in the region of the lesion at oneyear follow-up. This was thought to be a complication of the biopsy procedure and was treated by resection and proximal ulnar lengthening.
\end{abstract}

Florid reactive periostitis (FRP) is a benign bone-forming lesion that frequently involves the hands and feet of adolescents or young adults, and usually resolves spontaneously. ${ }^{1}$ The aetiology is unclear but a history of trauma is reported in about $40 \%$ of cases. ${ }^{1}$ There are only three previous reports of FRP in children, involving the tibia, ulna and femur, all of which resolved uneventfully. ${ }^{2-4}$ We report a child with FRP of both bones of the forearm which resolved with a small residual exostosis.

\section{Case report}

A ten-year-old girl was referred with a five-week history of painful swelling of her left wrist. There was no history of trauma, and pain preceded the swelling by one week. She had been treated with antibiotics and immobilisation of the wrist, but her symptoms had worsened. Initial examination showed a diffuse, tender, warm swelling of the dorsoulnar aspect of the wrist, with movement limited by pain. Laboratory investigations revealed a polymorph cell-predominant white blood cell of count $9.2 \times 10^{10} / 1$ and an ESR of $40 \mathrm{~mm} / \mathrm{h}$. Serum lactate dehydrogenase was $490 \mathrm{IU} / \mathrm{l}$ and alkaline phosphatase $182 \mathrm{IU} / \mathrm{l}$.

Radiographs revealed an irregularly lobulated mineralised lesion, measuring approximately $15 \mathrm{~mm} \times 13 \mathrm{~mm}$, between the distal radius and the ulna, and a circumferential periosteal reaction affecting the distal half of both forearm bones, mainly along the interosseous margins (Fig. 1). The distal radius was sclerotic. MRI confirmed the radiological findings and localised the lesion to the extensor compartment (Fig. 2). There was extensive T2 hyperintensity consistent with oedema, extending from the radiocar-

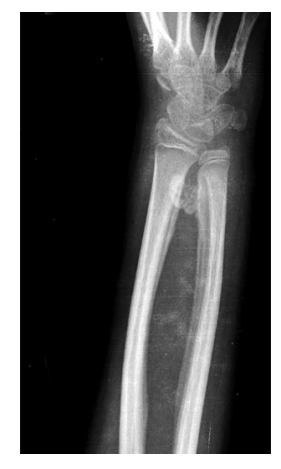

Fig. 1a

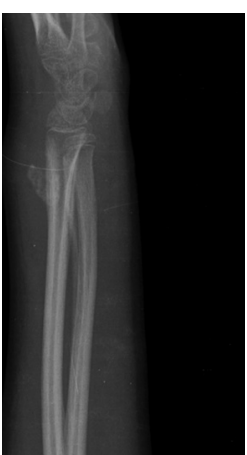

Fig. 1b
Initial radiographs showing sclerosis of the distal radius and ulna, circumferential periosteal reaction and a soft tissue calcified shadow in the distal forearm (a). The irregular soft tissue shadow is prominent dorsally (b).

pal joint to the proximal radioulnar joint and along the margins of the interosseous membrane (Fig. 2). Bone scintigraphy showed increased tracer uptake in the distal ulna.

A needle biopsy was inconclusive and an open biopsy was performed. A firm grey-white mass was found, and histology showed spindleshaped fibroblasts and irregular islands of woven bone (Fig. 3), with many osteoblasts (Fig. 4), confirming its reactive nature. In conjunction with the clinical and radiological findings, these features were considered diagnostic of FRP. Her wrist was immobilised for six weeks and she was given Naproxen $250 \mathrm{mg}$ twice daily. The swelling reduced, and at eight weeks' follow-up her wrist movements had improved. A radiograph at five months revealed a reduction in the size of the lesion (Fig. 5). One year later a 


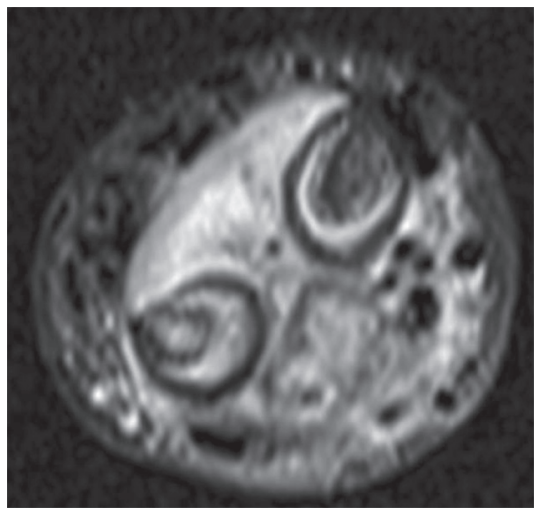

Fig. 2a

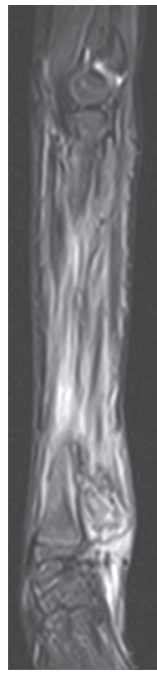

Fig. 2b
STIR axial (a) and sagittal (b) views of the forearm. The lesion is partly corticated, hyperintense, pushes the dorsal tendons laterally, and has caused significant oedema in the adjacent soft tissues in both compartments. Periosteal reaction and marrow changes are more obvious in the distal ulna.

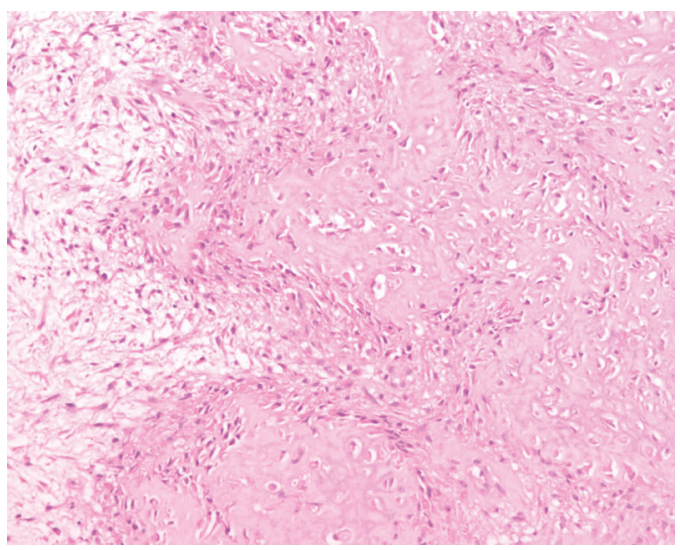

Fig. 3

Photomicrograph sshowing spindle-shaped fibroblasts adjacent to masses of woven bone (Haematoxylin \& eosin, $\times 100$ ).

small exostosis remained at this site, causing a peripheral physeal bar leading to angulation and $1 \mathrm{~cm}$ shortening of the ulna Fig. 6). Palmar- and dorsiflexion were full, but supination and pronation were limited to $50^{\circ}$ and $45^{\circ}$ respectively. The physeal bar and ulnar exostosis were excised and the ulna was lengthened by $1 \mathrm{~cm}$ using a minimal lengthener (Orthofix Srl., Verona, Italy) (Fig. 7). Further histological review revealed elements of physeal cartilage in the initial needle biopsy, suggesting that this was the cause of the physeal arrest.

\section{Discussion}

FRP was first described in 1981 by Spjut and Dorfman ${ }^{1}$ in a series of 12 patients where the small tubular bones of the hands and feet were involved. Although the term suggested

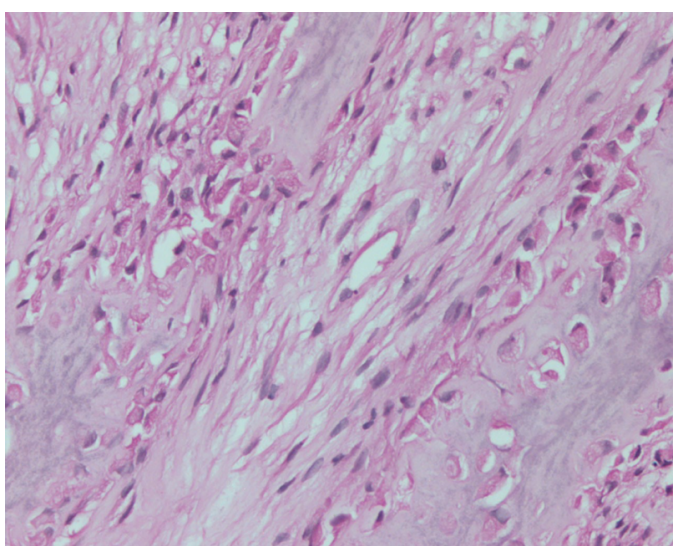

Fig. 4

Photomicrograph showing the bone lined by a layer of plump osteoblasts (Haematoxylin \& eosin, $\times 400$ ).

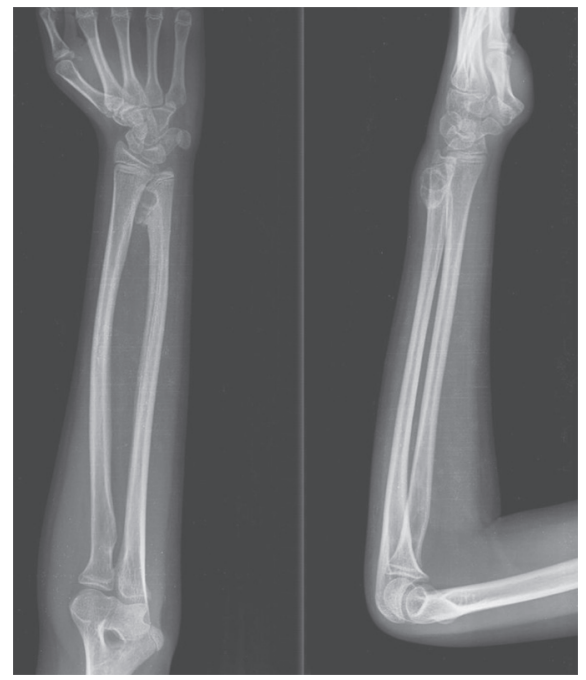

Fig. 5

Radiographs at five months from onset: the periosteal reaction is incorporated in the radius and ulna. The soft-tissue mass is now attached to the distal ulna, with the beginning of growth arrest.

by these authors is currently the most widely used, it is a misnomer because the condition is not a type of inflammation. It is a pronounced periosteal reaction, for which no obvious cause can be found. Histologically there is reactive proliferation of fibroblasts and osteoblasts, accompanied by new bone formation. It usually presents clinically as a painful swelling. It may be nodular or diffuse, and radiologically presents as a mineralised mass and periosteal reaction associated with intact underlying bone. The differential diagnosis includes neoplasms, such as parosteal osteosarcoma, Ewing's sarcoma and leukaemia, ${ }^{4}$ as well as reactive processes such as myositis ossificans or infection.

Unlike FRP, in parosteal osteosarcoma and Ewing's sarcoma the periosteal reaction is interrupted. In parosteal 


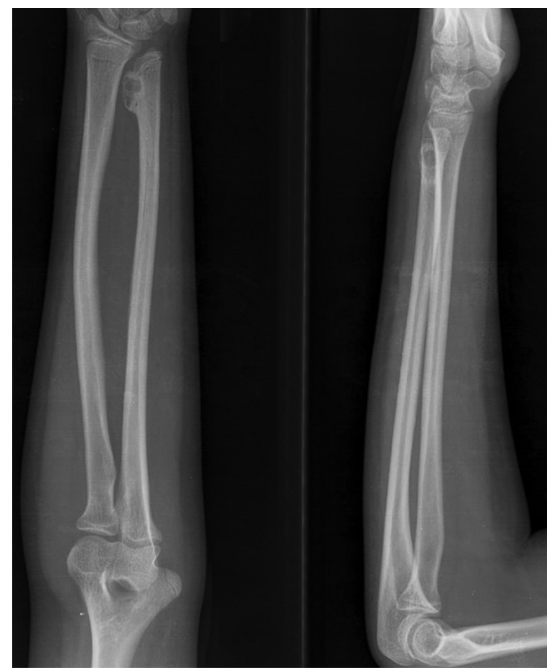

Fig. 6

Radiographs at 12 months after presentation showing a well-defined turret exostosis at the distal ulna, with shortening and radial tilt.
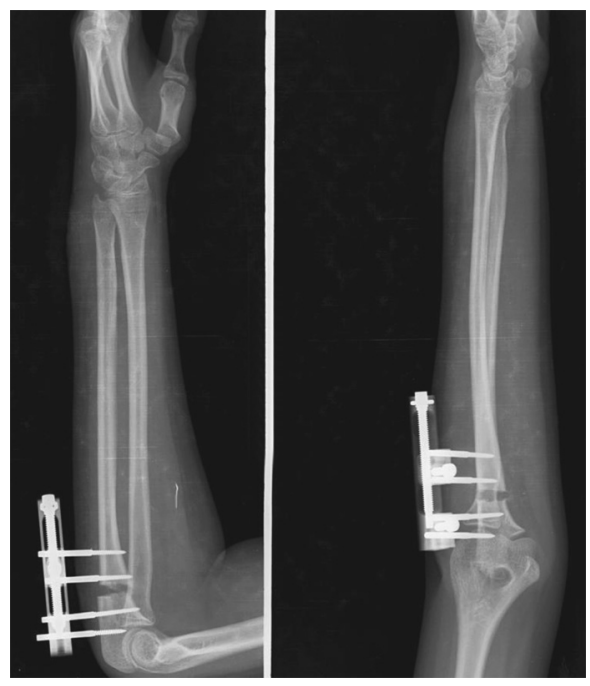

Fig. 7

Radiographs after excision of the turret exostosis and the peripheral physeal bar. Lengthening of the ulna using a minirail lengthener is in process.

osteosarcoma mineralisation extends to the periphery, and the zoning phenomenon that is sometimes seen in FRP is absent. ${ }^{5}$ Haematological malignancies can be differentiated by the presence of osteopenia, demineralisation in the metaphysiodiaphyseal region and radiolucent metaphyseal 'leukaemic' lines. ${ }^{6}$
Like FRP, myositis ossificans is also a reactive bone-forming mesenchymal proliferation. However, it is located in the muscle, and in its pure form usually lacks a pronounced periosteal reaction. Osteomyelitis is often associated with a marked periosteal reaction but there is usually also underlying bone resorption and radiolucencies. ${ }^{7}$ MRI can be used to differentiate FRP from marrow-based lesions such as osteomyelitis and Ewing's sarcoma, in children.

Dorfman has described a spectrum of reactive bone lesions and their progression with the passage of time, from FRP with minimal osteocartilaginous proliferation to a final mature ossified bony base with a cartilage cap known as turret exostosis. ${ }^{8}$ The exact time taken for this course has not been defined. In our patient, progression from the onset of symptoms to regression and periosteal remodelling took three months, to an ill-defined ossified mass six months, and to a distinct exostosis one year.

Physeal arrest has not been previously described as a complication in a paediatric patient with FRP, although all three previously described cases in the long bones involved the metaphysis and were close to the growth plate. ${ }^{2-4}$ Therefore, injury to the growth plate might occur, as in our patient secondary to biopsy. Care should therefore be taken to avoid the physeal plate when removing tissue for histological study.

Rapid growth of the distal radius resulted in a discrepancy of $1 \mathrm{~cm}$ within one year. In a child with more than two years' growth remaining, physeal surgery may be indicated. Our patient had more than five years of growth remaining and there was a progressive discrepancy in length between the radius and the ulna. The peripheral physeal bar was amenable to resection. These factors contributed to our decisions to simultaneously resect the physeal bar and the exostosis and lengthen the ulna.

No benefits in any form have been received or will be received from a commer cial party related directly or indirectly to the subect of this article.

\section{References}

1. Spjut HJ, Dorfman HD. Florid reactive periostitis of the tubular bones of the hands and feet: a benign lesion which may simulate osteosarcoma. Am J Surg Pathol 1981:5:423-33

2. Brien EW, Zahiri CA, Mirra JM. Florid reactive periostitis ossificans of the proximal aspect of the tibia: a lesion that must be distinguished from osteosarcoma. $J$ Bone Joint Surg [Am] 1999;81-A:1002-7.

3. Porcel López MT, Fernández Gil MA, Campos de Orellana A, Quiles Galindo M. Florid reactive periostitis ossificans of the distal ulna. Orthopedics 2008;31:286.

4. Azorín D, López-Pino MA, González-Mediero I, Epeldegui T, López-Barea F. Long bone florid reactive periostitis ossificans: a case in the distal femur mimicking osteosarcoma. J Pediatr Orthop B 2008;17:301-5.

5. Ackerman LV. Extra-osseous localized non-neoplastic bone and cartilage formation (so-called myositis ossificans): clinical and pathological confusion with malignant neoplasms. J Bone Joint Surg [Am] 1958;50-A:279-98.

6. Rogalsky RJ, Black GB, Reed MH. Orthopaedic manifestations of leukemia in children. J Bone Joint Surg [Am] 1986;68-A:494-501.

7. Lazzarini L, Mader J-T, Calhoun JH. Osteomyelitis in long bones. J Bone Joint Surg [Am] 2004;86-A:2305-18.

8. Dhondt E, Oudenhoven L, Khan S, et al. Nora's lesion: a distinct radiological entity? Skeletal Radiol 2006;35:497-502. 